Copyright C1997, American Institute of Aeronautics and Astronautics, Inc.

AIAA Meeting Papers on Disc, January 1997

A9715298, AIAA Paper 97-0222

\title{
Probability-based collision alerting logic for closely-spaced parallel approach
}

\author{
Brenda D. Carpenter \\ MIT, Cambridge, $M A$ \\ James K. Kuchar \\ MIT, Cambridge, $M A$
}

\begin{abstract}
AIAA, Aerospace Sciences Meeting \& Exhibit, 35th, Reno, NV, Jan. 6-9, 1997
A prototype airborne collision alerting logic was developed for aircraft on approach to closely-spaced parallel runways. A novel design methodology was used based on collision probabilities instead of traditional spatial or temporal alerting criteria. With this technique, an alert is issued when the probability of a collision exceeds an acceptable threshold value. The logic was based on a hazard level corresponding to the current Precision Runway Monitor System of one accident in every one thousand worst-case blunders. Probability contours were constructed through Monte Carlo simulation over a range of aircraft position, speed, heading, and turn rate conditions. These contours were stored in look-up tables that were accessed in real time for evaluation during numerical simulation of approaches. Three runway spacings were investigated: 3,400, 2,500, and $1,700 \mathrm{ft}$. The results show that the unnecessary alert rate at 1,700 ft runway spacing was double that at 3,400 ft runway spacing. Additionally, the logic induced collisions in two low-closure-rate situations, suggesting that the worst case blunder may not be a drastic heading change. (Author)
\end{abstract}


AIAA-97-0222.

\title{
PROBABILITY-BASED COLLISION ALERTING LOGIC FOR CLOSELY-SPACED PARALLEL APPROACH
}

\author{
Brenda D. Carpenter ${ }^{*}$ and James K. Kuchar ${ }^{\dagger}$ \\ Department of Aeronautics and Astronautics \\ Massachusetts Institute of Technology \\ Cambridge, MA 02139
}

\begin{abstract}
A prototype airborne collision alerting logic was developed for aircraft on approach to closely-spaced parallel runways. A novel design methodology was used based on collision probabilities instead of traditional spatial or temporal alerting criteria. With this technique, an alert is issued when the probability of a collision exceeds an acceptable threshold value. The logic was based on a hazard level corresponding to the current Precision Runway Monitor System of one accident in every one thousand worst-casc blunders. Probability contours were constructed through Monte Carlo simulation over a range of aircraft position, speed, heading, and turn rate conditions. These contours were stored in look-up tables that were accessed in real time for evaluation during numerical simulation of approaches. Three runway spacings were investigated: $3,400 \mathrm{ft}, 2,500 \mathrm{ft}$, and 1,700 ft. The results show that the unnecessary alert rate at $1,700 \mathrm{ft}$ runway spacing was double that at $3,400 \mathrm{ft}$ runway spacing. Additionally, the logic induced collisions in two lowclosure-rate situations, suggesting that the "worst case" blunder may not be a drastic heading change.
\end{abstract}

\section{Introduction}

Independent parallel approaches in Instrument Meteorological Conditions (IMC) can currently be conducted to runways as close as $3,400 \mathrm{ft}$ apart with the use of the Precision Runway Monitoring (PRM) System. ${ }^{1-6}$ PRM uses a high-resolution ground radar to provide a depiction of the approach situation to an air traffic controller. The controller monitors the aircraft on approach relative to a No Transgression Zone (NTZ) between the runways (Figure 1). When an aircraft strays into the NTZ (termed a blunder), the controller issues break-out instructions to prevent a collision.

A major limitation of PRM is the fact that there can be significant time delays between when an aircraft begins to transgress, when a controller observes that

* Graduate Rescarch Assistant, Member AIAA

${ }^{\dagger}$ Assistant Professor, Member AIAA

Copyright $\bigcirc 1997$ by MIT. Published by the American Institute of Aeronautics and Astronautics, Inc. with permission. transgression, and when the controller is able to clear the frequency and issue breakout instructions. To provide adequate safety, the NTZ must be relatively large and is $2,000 \mathrm{ft}$ wide.

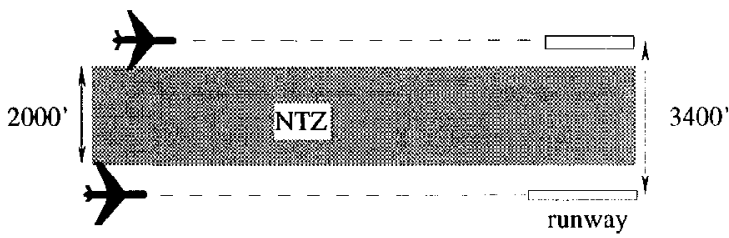

Fig. 1 Parallel Approach with No Transgression Zone (NTZ)

Because of the potential for large time delays, PRM has been estimated to be unable to resolve approximately 1 in 250 "worst-case blunders" (defined as a sudden $30^{\circ}$ heading change intrusion by one aircraft). ${ }^{6}$ When runways are spaced closer than $3,400 \mathrm{ft}, \mathrm{PRM}$ is unable to provide this safety level without producing an excessive number of false alarms. ${ }^{5}$ Also, at close runway spacings, a spatial NTZ cannot provide enough lead time to prevent a collision. Position derivatives such as closure rate or acceleration are required to detect a blunder in time to successfully resolve it.

An airborne alerting system would have the benefit of eliminating much of the time delay by warning the flight crews directly. However, current airborne alerting systems such as the Traffic Alert and Collision Avoidance System (TCAS) would produce an unacceptable false alarm rate at runway spacings closer than approximately 3,000 $\mathrm{ft}^{7}$ This is because TCAS has been designed for enroute and terminal area maneuvering operations and is not optimized for flight in close proximity to other aircraft. Thus, a specialized airborne alerting logic is required if independent parallel approaches are to be conducted in IMC to runways less than $3,400 \mathrm{ft}$ apart.

To meet this need, and to serve as a testbed for examining research issues, a prototype airborne alerting logic was developed for closely-spaced parallel approach. 
The logic uscs datalinked aircraft state information (position, velocity, heading, turn rate) to determine if an alert is needed. The primary design goal was to maintain current PRM safety levels while keeping an acceptable nuisance alarm rate. This paper describes the development of the prototype alerting logic and its subsequent evaluation in simulation studies.

\section{Development of Alerting Logic}

To simplify its development, the prototype alerting logic focuses on the final approach segment when both aircraft are cstablished on the approach path, and assumes that the aircraft are coaltitude until an alert is generated. Runway stagger and the turn onto the localizer are ignored. Also, the large-scale traffic management of aircraft in the terminal area is not considered.

In contrast to current alerting systems (e.g., PRM or TCAS), the logic described here does not use a standard spatial or temporal (time to impact) alerting criterion. Instead, the logic bases the alerting decision on the estimated probability of a collision. Thus, alerts are issued at a consistent level of safety rather than, for example, at a consistent time before impact.

Because the logic is based on the probability of collision, it was necessary to develop a dynamic model of aircraft on approach that included consideration of uncertainties in sensor measurements and in the intentions of the aircraft. A series of Monte Carlo simulations were then used to estimate the probability of a collision as a function of aircraft position, speed, heading, and turn rate. Alerting thresholds were designed to correspond with a specified probability of collision and were stored in a series of look-up tables. In operation, the logic compared measurements of aircraft state against the look-up tables and issued an alert if the estimated probability of collision was above the threshold.

\section{Dynamic Models}

An analysis of the dynamics of approaches and blunders was conducted to determine the importance of having access to information about aircraft states such as relative position, heading, and turn ratc. Estimates of these parameters are assumed to bc availablc onboard the aircraft through datalink such as Automatic Dependent Surveillance - Broadcast (ADS-B) or through a measurement filtering technique. It was determined that knowledge of the relative position, speed, heading, and turn rate (or bank angle) of parallel traffic greatly enhances the ability to determine whether a situation is hazardous. Higher derivatives such as rate of change of bank angle are generally too noisy to produce reliable projections into the future.

Because all mcasurements contain noise, the alerting system must decide whether a given measurement indicates a potential blunder or whether it results from normal oscillations on the approach path. The alerting system must therefore balance the probability of a missed or late detection against the probability of an unnecessary alert. To view this tradeoff directly, a probabilistic approach was taken.

Figure 2 shows the potential future flight paths of a blundering intruder currently in a left turn. Based on an estimate of aircraft heading and bank angle, the aircraft is projected to fly a certain predicted trajectory. However, because of uncertainties in the state estimate and because the intruder may modify its maneuver, the actual path followed by the intruder may be different (shown as a shaded region). The size and shape of the shaded region are determined from a probabilistic dynamic model.

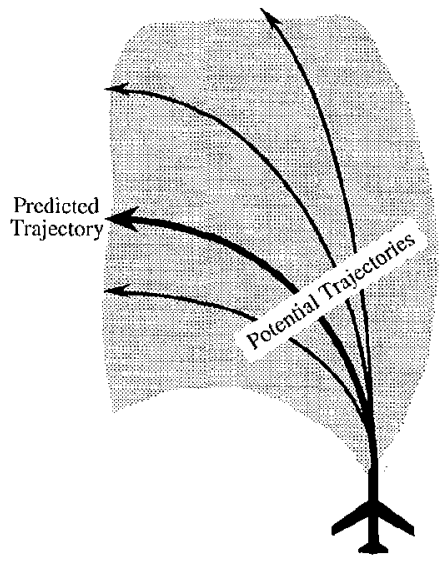

\section{Fig. 2 Potential Intruder Trajectories}

In the dynamic model, the intruding aircraft is nominally projected to fly a constant-rate turn at constant altitude based on its measured position, heading, speed, and turn rate. To account for uncertainties in this projection, random errors are introduced into the starting conditions during Monte Carlo simulation. In the dynamic model, the position estimate of the aircraft is modeled as a zero-mean normally-distributed random variable with standard deviation of $35 \mathrm{ft}$ (corresponding to Differential GPS accuracy). Aircraft heading and bank angle are modeled with standard deviations of $2.5^{\circ}$ and $5^{\circ}$ respectively. The magnitudes of these uncertainties were determined through analysis of aircraft state data during simulated approaches in varying turbulence conditions. 
When an alert is issued by the prototype system, the threatened aircraft is assumed to fly a specified avoidance maneuver consisting of a climbing turn away from the parallel traffic. Following a two-second response delay from the alert, a $0.25 \mathrm{~g}$ pull-up mancuver and a $45^{\circ}$ turn at a $30^{\circ}$ bank angle is performed.

\section{Monte Carlo Simulation}

The models of the intruding aircraft and of the threatened aircraft's avoidance maneuver were used in a series of Monte Carlo simulations to determine the probability of a collision over a range of conditions. By numerically extrapolating the positions of the two aircraft on approach, it can be determined whether a collision (defined as separation less than $500 \mathrm{ft}$ ) will occur in a given situation. The probability that a collision will occur is then estimated by counting the number of collisions that occur over a large number of Monte Carlo simulations.

Monte Carlo simulations were performed at each of a number of initial conditions specified by aircraft position, speed, heading, and bank angle. To each initial condition were added the random errors described in the previous section, and the aircraft's trajectories were numerically simulated to determine if a collision occurred.

Table 1 shows the set of initial intruder conditions used in the Monte Carlo simulations. Initial intruder position was varied within a grid with dimensions of $4,400 \mathrm{ft}$ laterally and extending $9,200 \mathrm{ft}$ behind to 9,200 $\mathrm{ft}$ ahead of the threatened aircraft. Grid points were placed every $400 \mathrm{ft}$ in this region. Intruder speed was set from 120 - $180 \mathrm{kts}$, in increments of $20 \mathrm{kt}$. Aircraft heading was set from $40^{\circ}$ away to $40^{\circ}$ toward the threatened aircraft in increments of $10^{\circ}$. Finally, intruder bank angle was set from $20^{\circ}$ away to $40^{\circ}$ toward the threatened aircraft in increments of $10^{\circ}$. The total number of combinations of states in Table 1 results in
142,128 different conditions. At each condition, the random errors described previously were introduced and the aircraft were simulated to determine if a collision occurred. 10,000 Monte Carlo runs were performed at each condition.

The result of these Monte Carlo simulations is a multidimensional map that describes the probability of a collision as a function of intruder position, speed, heading, and bank angle. The result applies only to the specific avoidance maneuver performed by the threatened aircraft: a different map is generated for different actions by the threatened aircraft. This map is best visualized by using probability contours. Figure 3 shows a schematic of the probability contours for a single combination of intruder speed, heading, and bank angle, and assuming that the threatened aircraft performs the climbing turn avoidance maneuver. A different set of contours was constructed for each combination of intruder speeds, headings, and bank angles in Table 1.

In Figure 3, the threatened aircraft is shown in black at the origin of the coordinate system. The probability of a collision, as determined from the Monte Carlo simulations, is marked in the figure using contours. Example intruders are shown in white at positions $\mathbf{A}$, $\mathbf{B}$, and $\mathbf{C}$. As a reference, if an intruder is located on the dark curved line, it is projected to have a direct collision with the threatened aircraft if it does not change its speed or turn rate.

\section{Alerting Threshold Definition}

The alerting threshold was designed to correspond to a constant probability of collision. This probability was set at $p=0.001$, which is the same order of magnitude as the PRM system. ${ }^{6}$ Thus, in Figure 3 the extent of the alerting threshold is defined by the $\mathbf{p}=0.001$ contour. Because the shape of this contour varies depending on the intruder's state, the alerting threshold's spatial extent also varies.

Table 1 Intruder State Initial Conditions

\begin{tabular}{ccc}
\hline State & Extent & Increments \\
\hline Lateral Position & $0 \mathrm{ft}-4,400 \mathrm{ft}$ & $400 \mathrm{ft}$ \\
Longitudinal Position & $9,200 \mathrm{ft}$ behind $-9,200 \mathrm{ft}$ ahead & $400 \mathrm{ft}$ \\
Speed & $120 \mathrm{kt}-180 \mathrm{kt}$ & $20 \mathrm{kt}$ \\
Heading & $40^{\circ}$ away $-40^{\circ}$ toward threatened aircraft & $10^{\circ}$ \\
Bank Angle & $20^{\circ}$ away $-40^{\circ}$ toward threatened aircraft & $10^{\circ}$ \\
\hline \hline
\end{tabular}




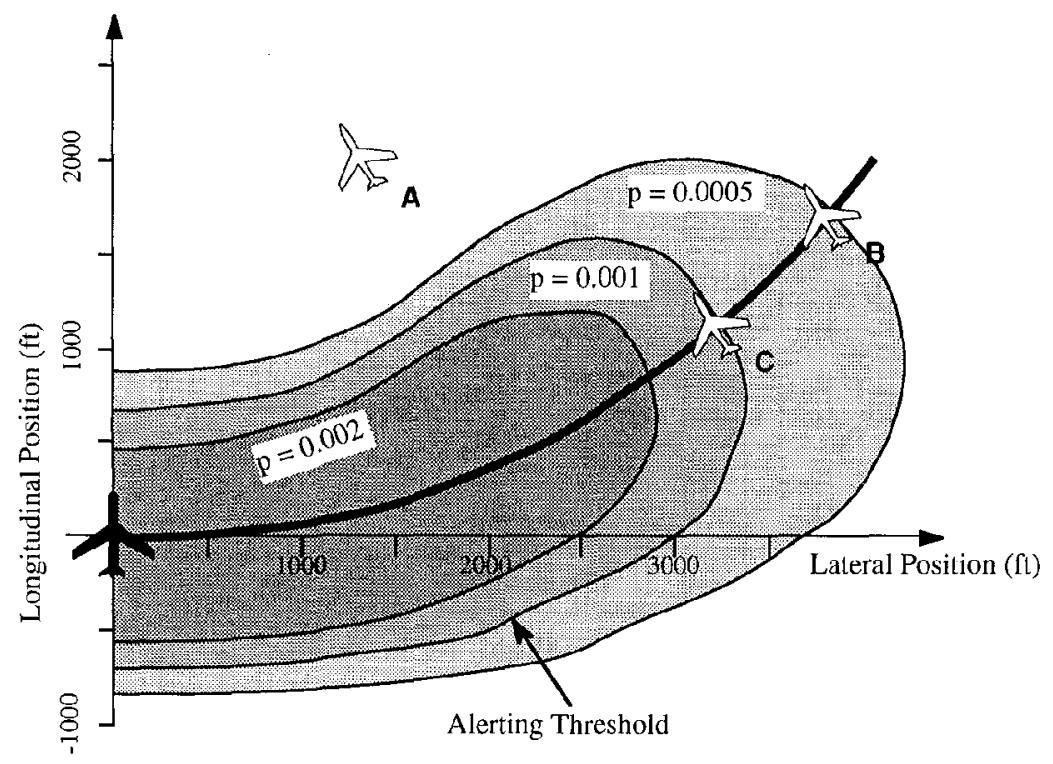

Fig. 3 Example Probability of Collision Contours (Schematic)

In the example situation shown in Figure 3, an intruder in position $\mathbf{A}$ does not trigger an alert because it has to greatly decrease its speed or increase its turn rate to cause a collision. An aircraft in position $\mathbf{B}$ is also outside the threshold and does not trigger an alert: although the intruder is projected to collide with the threatened aircraft, time still remains with which to delay the alert before the probability of a collision reaches the threshold value of $p=0.001$. An aircraft at C generates an alert because the probability of a collision has risen above the threshold. Similar plots and alerting thresholds were developed at each combination of speed, heading, and bank angle from Table 1.

Calculation of the probability of collision in real time is not feasible for the types of processors currently available on aitcraft. Accordingly, it was necessary to store the shape and size of the alerting threshold rather than calculate it explicitly during operation. To facilitate storage and processing, the alerting threshold shapes were simplified so that they could be accessed rapidly in real time.

Two criteria were used to define the shape and size of the alerting thresholds. First, the intruder must be projected to pass within $800 \mathrm{ft}$ of the threatened aircraft. The value of $800 \mathrm{ft}$ corresponds approximately to the longitudinal width of the $p=0.001$ contours rclative to the curved direct collision line. Second, the intruder must be within a certain range of the threatened aircraft (corresponding to the lateral extent of the $p=0.001$ contour). This range parameter is also a function of airspeed, heading, and bank angle. If both tests are passed, an alert is issued.

The alerting threshold parameters were codified in a series of look-up tables for real-time access. In use, the logic takes a set of state measurements and compares them to the look-up table parameters to determine if an alert should be issued. Parameter values werc interpolated between tables when the intruder states varied between the conditions in Table 1 . The alcrting algorithm was implemented in $\mathrm{C}$ code and used in the MIT part-task Advanced Cockpit Simulator and in parttask simulations at the NASA Ames Research Center. $^{9.10}$

\section{Evaluation of the Logic}

The performance of the prototype alcrting logic was evaluated using different approach trajectories developed from flight simulation tests at Rockwell-Collins. ${ }^{8}$ These included normal approaches and six categories of blunder trajectories: a slow constant-rate turn at a $5^{\circ}$ bank angle; heading-changes of $10^{\circ}, 15^{\circ}, 30^{\circ}$; and two cases in which the intruder began a blunder but returned to its approach path before crossing the threatened aircraft's approach path. Separate trajectory data were available for calm and turbulent conditions and at airspeeds of 130,145 , and $160 \mathrm{kt}$. The same Irajectories were used at three runways spacings $(1,700 \mathrm{ft}, 2,500 \mathrm{ft}$, and $3,400 \mathrm{ft}$ ) and over a series of initial longitudinal spacings (within $\pm 1.5 \mathrm{nmi}$ ) to cover a range of possible

4

American Institute of Aeronautics and Astronautics 
Table 2 Outcome Categories

\begin{tabular}{cccc}
\hline \hline Outcome Category & Alcrt Issucd? & Collision Occurred? & Alert Necessary? \\
\hline Correct Rejection & No & No & No \\
Missed Detection & No & Yes & Yes \\
Unnecessary Alert & Yes & No & No \\
Induced Collision & Yes & Yes & No \\
Correct Detection & Yes & No & Yes \\
Late Alert & Yes & Yes & Yes \\
\hline \hline
\end{tabular}

encounter situations. A total of 42,822 simulations using 39 different types of trajectories were performed for the evaluations.

\section{Procedure}

In the evaluations, the threatened aircraft followed a normal approach path while the intruder followed one of the blunder or normal approach paths described previously. The alerting logic was implemented and if an alert was issued, the threatened aircraft performed the specified climbing-turn avoidance maneuver. The outcome of each approach was recorded, including (1) whether an alert was generated, (2) whether a collision occurred, and (3) whether an alert was deemed necessary. Six categories were used to define the possible outcomes, listed in Table 2. A collision was defined to occur if separation at any point in the approach was less than $500 \mathrm{ft}$. An alert was considered to be necessary if a collision would have occurred without an alert. Thus, for example, an alert in a situation in which separation would have been $501 \mathrm{ft}$ without the alert was categorized as unnecessary. Even though such an alert could be considered to be warranted, a specific definition of unnecessary alert is required as a performance metric.

From Table 2, if an alert was not issued at any time during a run it was classified as either a Correct Rejection (if a collision did not occur) or as a Missed Detection (if a collision did occur). If an alert was issued, the outcome was placed in one of four categories. An Unnecessary Alert was a case where the intruder was not on a direct collision course, an alert was issued anyway, and a collision was still avoided. If a collision occurred because of the alert, it was classified as an Induced Collision. A Correct Detection occurred when a collision was averted because of an alcrt. Finally, a Late Alert was a case in which an alert was issued but was too late to prevent a collision.

\section{Results}

The results of the evaluations are compiled in Table 3 as the observed rate of occurrence of each of the six possible outcomes at each of the three runway spacings. It must be stressed that the observed rates are dependent on the mix of the specific types of blunder scenarios used in the evaluations and are not indicative of the expected rates during a typical approach. Thus, the values in Table 3 are useful as indicators of performance but are not absolute measures.

In those scenarios in which the intruder followed a normal approach trajectory and did not blunder, no alerts were issued. Alerts were only issued in sccnarios in which a blunder occurred. Therefore, it appears that the logic was able to distinguish between nominal approach oscillations and blunders down to $1,700 \mathrm{ft}$ runway spacing. However, a more complete study of aircraft on approach is required to verify this finding.

As shown in Table 3, there were no Missed Detections or Latc Alcrts at any runway spacing. This indicates that alerts, when necessary, were issued early enough that collisions could be avoided. A more complete safety assessment would require modeling the probability that the system fails to operate as designed (e.g., because of loss of datalink between aircraft).

Table 3 Logic Performance

(Rates based on 14,274 simulations at each runway spacing)

\begin{tabular}{ccccccc}
\hline $\begin{array}{c}\text { Runway } \\
\text { Spacing }\end{array}$ & $\begin{array}{c}\text { Correct } \\
\text { Rejections }\end{array}$ & $\begin{array}{c}\text { Missed } \\
\text { Detections }\end{array}$ & $\begin{array}{c}\text { Unnecessary } \\
\text { Alerts }\end{array}$ & $\begin{array}{c}\text { Induced } \\
\text { Collisions }\end{array}$ & $\begin{array}{c}\text { Correct } \\
\text { Detections }\end{array}$ & $\begin{array}{c}\text { Late } \\
\text { Alerts }\end{array}$ \\
\hline $1700^{\prime}$ & $9.1 \times 10^{-1}$ & 0 & $4.7 \times 10^{-2}$ & $4.2 \times 10^{-4}$ & $3.8 \times 10^{-2}$ & 0 \\
$2500^{\prime}$ & $9.4 \times 10^{-1}$ & 0 & $3.0 \times 10^{-2}$ & $4.2 \times 10^{-4}$ & $3.2 \times 10^{-2}$ & 0 \\
$3400^{\prime}$ & $9.5 \times 10^{-1}$ & 0 & $2.3 \times 10^{-2}$ & 0 & $3.2 \times 10^{-2}$ & 0 \\
\hline \hline
\end{tabular}


These effects could result in Missed Detections or Late Alerts but were not modeled in this evaluation.

Some situations at $1,700 \mathrm{ft}$ and $2,500 \mathrm{ft}$ runway spacings resulted in Induced Collisions. That is, there were some cases during a blunder in which an alert calsed the collision. Had the system not alerted, there would not have been a collision. These Induced Collisions occurred only in the $5^{\circ}$ bank angle and $10^{\circ}$ heading change blunder trajectorics, suggcsting that the alerting logic is deficient when the closure rate between aircraft is relatively low. This deficiency is likely due to the approximations used to develop the alerting thresholds, and it is believed that the logic can be modifjed to reduce or eliminate the Induced Collision rate.

No collisions occurred in any of the other blunder scenarios, including the traditional "worst-case" $30^{\circ}$ turn blunder. Thus, if the $30^{\circ}$ blunder is used as the criterion for safety (as it is for PRM), then the prototype logic performs well down to $1,700 \mathrm{ft}$ runway separation. The fact that some collisions did occur in the more benign blunder cases points to the important fact that the "worst case" scenario for a given system may not be the trajectory that appears at first to be the most threatening. A gradual closure-rate scenario may in fact be more dangerous with some algorithms.

Some of the alerts, as shown in Table 3, were categorized as Unnecessary. These Unnecessary Alerts were cases in which a collision did not occur, but a collision would also not have resulted had the alert not been issued (i.e., the intruder was not on a collision course). Unnecessary Alerts occurred in increasing proportion as the runways were placed closer together. At $1,700 \mathrm{ft}$ runway spacing, the observed Unnecessary Alert rate is more than double that at $3,400 \mathrm{ft}$.

Recall that as defined here, an Unnecessary Alert is a case in which morc than $500 \mathrm{ft}$ separation would have existed had no alert been issued. Figure 4 shows the distribution of miss distances had no alerts been issued (for the Unnecessary Alert cases only). In the figure, the majority of Unnecessary Alerts were such that the miss distance would have been less than $1,000 \mathrm{ft}$. At 2,500 ft runway spacing, for example, $97 \%$ of all Unnecessary Alert situations would have resulted in less than 1,000 $\mathrm{ft}$ separation had the alert not been issued. Thus, although Unnecessary Alerts were relatively common, many of them could be considered to be warranted because miss distances of less than $1,000 \mathrm{ft}$ would have occurred.

Note that there is a significant change in the miss distance distribution between $1,700 \mathrm{ft}$ and the larger two runway spacings. While $97 \%$ of the cases at $2,500 \mathrm{ft}$ runway spacing would have resulted in less than 1,000 ft miss distances, at $1,700 \mathrm{ft}$ runway spacing this rate drops to $75 \%$. This indicates that the alerting logic begins to have difficulty in rejecting Unnecessary Alerts as runway spacing decreases below $2,500 \mathrm{ft}$.

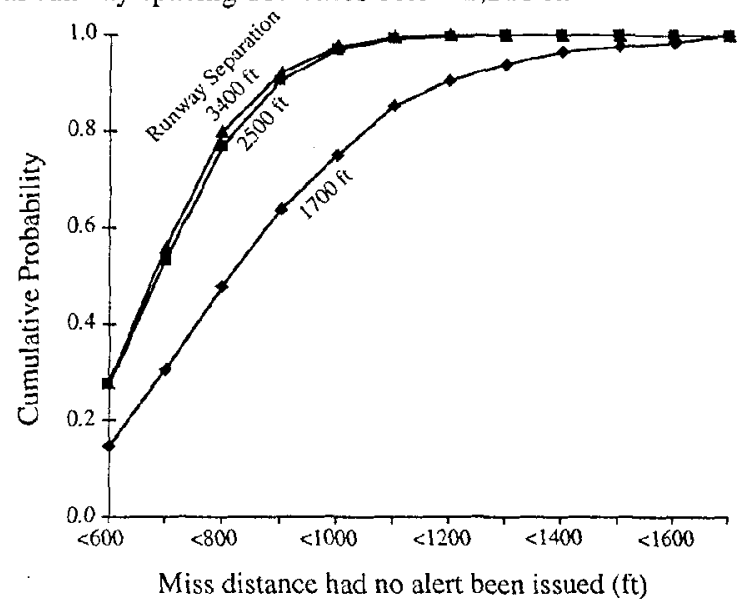

Fig. 4 Miss Distance Distribution During Unnecessary Alerts

Again, it must be stressed that all Unnecessary Alerts occurred during blunder scenarios and therefore they all could be considered to be warranted. No alerts occurred during normal approach scenarios at any runway spacing.

\section{Additional Considerations}

The alerting logic presented here is generally effective in the cases that were studied because the climbing component of the avoidance maneuver provides vertical separation. If the intruder also climbs, however, additional collisions could result because this vertical separation would be reduced or eliminated. The incorporation of vertical state information (relative altitude and vertical rate) will be necessary in an operational system. These additional states will be required both to improve safety in cases in which the intruder climbs and also to aid in rejecting alerts when the intruder is clcarly above or below the threatened aircraft's flight path.

An additional issue regards the availability of alternative avoidance maneuvers such as level turns or straightahead climbs. The ability to select one of several avoidance strategies could enhance the performance of the alerting systcm. For example, in a case in which the intruder climbs during a blunder, it may be more effective to command the threatened aircraft to perform a turn at constant altitude (or even to continue the 
approach) rather than climb. One issue that must be examined, however, is the potential impact that multiple maneuvers could make on pilot response. Pilots may be able to react more quickly to an alert when their response is known beforehand. When the pilot must perform one of several alternative actions in response to an alert (either through choice or by command), response time may increase, thereby reducing the intended benefit of providing multiple maneuver options. A related issuc is that the consistency with which pilots can fly a prescribed avoidance maneuver will impact system performance. In the evaluations conducted here, the avoidance maneuver was followed perfectly. In actuality, this will not be the case and some scatter in pilot response will occur.

One final issue involves the ability of the pilot to understand the underlying alert logic. Although a probability-based alerting logic may be more effective than a simpler spatial (NTZ) method, it may be more difficult for a pilot to understand why alerts occur. As a result, pilots may distrust the system, reducing its effectiveness.

\section{Conclusions}

A prototype airborne alerting logic for closely-spaced parallel approach has been designed and evaluated. A novel design methodology was applied in which the probability of a collision was directly used to set alerting thresholds. This approach contrasts with conventional design methods in which alerting thresholds are based on spatial or temporal (time to impact) criteria.

The logic was evaluated through numerical simulation of a variety of blunder and normal approach situations. This allowed a wide range of encounters to bc cxamined but these encounters may not be representative of actual operations. Statistical data on the frequency and geometry of blunders is not currently available, so it is difficult to assess the safety or unnecessary alert rate of a proposed system.

For the encounters used in the evaluations, no Unncccssary Alcrts were generated during normal approaches. This suggests that the logic performs well in rejecting alerts due to nominal tracking oscillations. The only Unnecessary Alerts that were observed occurred in blunder situations in which the minimum scparation would generally have been less than 1,000 ft. The Unnecessary Alert rate at $1,700 \mathrm{ft}$ runway separation was twice the rate at $3,400 \mathrm{ft}$ runway separation. However, the definition of "unnecessary" is arbitrary and must be considered when evaluating the results. An alert could be considered nccessary if any type of blunder occurs. Alternatively, as assumed here, an alert could be considered necessary only if it is required to avoid a collision.

Several collisions were induced by the alerting logic. These collisions occurred in low-closure-rate situations, and it is believed that the collision rate can be reduced through modifications to the alerting logic. However, the fact that the traditional "worst case" $30^{\circ}$ blunder was resolved more effectively than more benign blunders indicates that the "worst case" may not always be obvious.

Additional enhancements to the logic are required, including incorporation of altitude-related alerting criteria. Bccausc the current logic uses a climbing-turn avoidance maneuver, more collisions are expected in situations in which the intruder is also climbing. Altitude criteria are also required to reject alerts in cases in which the intruder is clearly above or below the threatened aircraft's flight path.

It may also be necessary for the logic to select one of several avoidance maneuvers (e.g., climbing turn, level turn, or straight climb). Each of these avoidance maneuvers can be examined in terms of probability contours similar to those used for this study. The contours could then be used to determine which maneuvers have the highest probability of success. There are several interesting design issues for systems in which multiple avoidance maneuvers are available. For example, a conservative system design could alert when any one of several avoidance maneuvers becomes unsafe duc to an intruder. An alternative design could delay alerting until only one of the avoidance options remains. The former system allows the pilot more latitude in determining the best action but will have more unnecessary alerts than the latter system, in which the pilot must accurately perform the single prescribed avoidance maneuver.

The probabilistic approach taken in the development and evaluation of the alerting logic can be applied to evaluate other types of alerting systems. For example, a spatial No Transgression Zone alerting system can be evaluated in terms of the probability of collision or unnecessary alert rate. Thus, when alerting methods ane proposed for closely-spaced parallel approach, the methodology used here can be applied in evaluating their performance.

A complete safety analysis of a proposed system for closely-spaced parallel approach will require estimates of the likelihood and composition of blunders. This information is not currently available, so the overall impact of an airborne alerting system on safety cannot 
be determined. Second, it will be important to evaluate the ability of a system to reject alerts during normal approaches. Given that GPS-guided approaches arc presumed in concepts for closely-spaced parallcl approach, it will be necessary to incorporatc data regarding aircraft tracking performance using GPS (e.g., lateral deviation, heading, and bank angle variability). Third, the impact of alcrts on the overall traffic flow must be examined. At this point, the alerting logic only provides protection for the immediate collision hazard but does not aid the pilot in returning into the approach sequence.

Finally, the issues raised during this study are important not only for closely-spaced parallel approaches, but apply to alerting system design in general. As advanced alerting systems are proposed for Free Flight or terrain avoidance, for example, issues such as the tradeoff between single or multiple response options will arise. Thus, there is a need to further develop gencric models of alerting that can be applied to different systems.

\section{Acknowledgment}

This research was supported by the NASA Langley Research Center through the MIT Lincoln Laboratory.

\section{References}

1 Federal Aviation Administration, "Precision Runway Monitor Demonstration Report", Document DOT/FAA/RD-91/5, February, 1991.

${ }^{2}$ Ebrahimi, Y. S., "Parallel Runway Requirement Analysis Study", NASA CR-191549, Vols. 1 \& 2, December 1993.

${ }^{3}$ Gladstone, C. X., "Overview of the Simultaneous Instrument Approach Model (SIAM) Version 1.0", MITRE Document No. MTR-94W0000074, June, 1994.

${ }^{4}$ Massimini, S. V., "The Blunder Resolution Performance Model", MITRE Working Paper WP91W00147, September, 1991.

${ }^{5}$ Owen, M. R., "The Memphis Precision Runway Monitor Program Instrument Landing System Final Approach Study", Lincoln Laboratory Report ATC-194, Document DOT/FAA/NR-92/11, May 23, 1993.

' Shank, E. M., and K. M. Hollister, "A Statistical Risk Assessment Model for the Precision Runway Monitor System", ATCA Conference Proceedings, 1992.

${ }^{7}$ Folmar, V. A., Szebrat, X. P., and N. E. Toma, "An Extension to the Analysis of Traffic Alert and Collision Avoidance System (TCAS) Advisories During Simultaneous Instrument Approaches to
Closely Spaced Parallel Runways", MITRE Document MTR-94W0000056, July, 1994.

${ }^{8}$ Chamberlain, S., and S. Koczo, "Close Parallel Runway Operations (CPROPS) Prototype Alerting System Definition and Evaluation", RockwellCollins Draft Report Paper, August 14, 1995.

${ }^{9}$ Pritchett, A., Carpenter, B., Asari, K., Kuchar, J. K., and R. J. Hansman, "Issues in Airborne Systems for Closely-Spaced Parallel Runway Operations", Proceedings of the 14th AIAA/IEEE Digital Avionics Systems Conference, Cambridge, MA, November, 1995.

${ }^{10}$ Carpenter, B., and J. K. Kuchar, "Prototype Alerting System Logic for Closely-Spaced Parallel Approach", MIT Aeronautical Systems Laboratory Report ASL-95-6, December 28, 1995. 